\title{
Fast further purification of diastereomeric salts of a non-racemic acid by gas antisolvent fractionation
}

\author{
Márton Körösi[ ${ }^{[b]}$, János Madarász ${ }^{[b]}$, Tamás Sohajda[c], and Edit Székely*[a]
}

\begin{abstract}
A novel, green possibility of the further purification of the diastereomeric salt of 4-chloromandelic acid and 1-phenylethane-1-amine has been developed. Gas antisolvent method using supercritical carbon dioxide was applied for the first time to precipitate the diastereomeric salts with increased purity followed by the supercritical fluid extraction of the dissolved diastereomers. The $R R$-salt can be purified to $>99 \%$, while fractionation based purification of the $S R$-salt is limited to approx. $80 \%$. The limiting initial diastereomeric excess correlates strongly to the atmospheric melting eutectic composition of the same
\end{abstract} salts which suggests that despite the fast precipitation, the diastereomeric excess of the solid product is not kinetically determined. The efficiency of the diastereomeric enrichment is in the same range as that of the atmospheric reference experiments, however, technological advantages provided by the antisolvent precipitation method such as fast processing and dry product obtained, suggest that this novel procedure is a promising alternative of the atmospheric methods.

Keywords: supercritical carbon dioxide; diastereomeric salt; enantiomeric enrichment; eutectic behaviour; melting point

Introduction

The high demand for enantiopure compounds in the pharmaceutical, agrochemical (etc.) industries makes it necessary to evaluate and improve both traditional and innovative methods of optical resolution and further purification of nonracemic mixtures. It was already shown that some methods involving high pressure (or supercritical) carbon dioxide, known as an environmentally benign and GRAS (generally recognized as safe) solvent, can be very effective in enantiomeric separations. ${ }^{1-}$ ${ }^{3}$ Supercritical techniques are typically well controllable, taking the advantages of fast diffusion, low viscosity and the controllable density of the solvent. Meanwhile, due to the high pressure required $(>7.3 \mathrm{MPa})$ investment costs are rather large and the safety issues must be addressed. One of the carbon dioxide employing methods is gas antisolvent (GAS) fractionation (FIGURE 1). ${ }^{4,5}$ GAS fractionation is performed in a high pressure vessel. A nearly saturated solution of the material to be precipitated (the diastereomeric salts in this case) is filled into the autoclave (FIGURE $1 \mathrm{a}$ )).

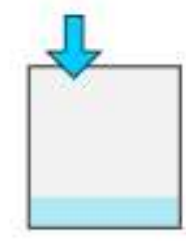

a)

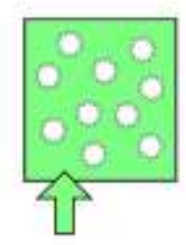

b)

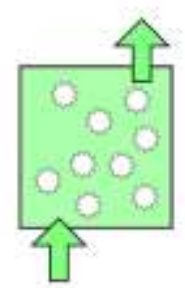

c)

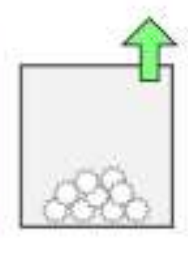

d)
The added carbon dioxide is dissolved in the organic solvent. The polarity thus the solvent power of the forming solvent mixture is significantly lower than that of the organic solvent, resulting in large oversaturation and fast precipitation (FIGURE 1 b)). By optimizing the operating conditions, partial precipitation can be achieved. The undesired, more soluble fraction and the organic solvent is extracted using carbon dioxide (FIGURE $1 \mathrm{c}$ )). After depressurisation the solid dry product is collected (FIGURE 1 d)).GAS and related methods have been widely demonstrated to be capable of producing fine powders with controlled particle size, varied morphology and low remaining organic solvent residues. ${ }^{6-}$ 9

[a] Associate professor, Dr., Székely

Department of Chemical and Environmental Process Engineering

Budapest University of Technology and Economics

Müegyetem rakpart 3.; $\mathrm{H}-1111$ Budapest

E-mail: $\underline{\text { sz-edit@mail.bme.hu }}$

[b] PhD. fellow; Körösi

Department of Chemical and Environmental Process Engineering

Budapest University of Technology and Economics

Müegyetem rakpart 3.; H-1111 Budapest

[b] Associate professor, Dr., Madarász

Department of Inorganic and Analytical Chemistry

Budapest University of Technology and Economics

Müegyetem rakpart 3.; $\mathrm{H}-1111$ Budapest

[c] Dr., Sohajda

Cyclolab Ltd.

Budapest, P.O.Box 435, H-1525 
The first detailed study of the GAS technique for the purification of diastereomeric salts is presented in this paper on the example of 1-phenylethanaminium - 4-chloromandelate diastereomeric salt. 4-chloromandelic acid is a chiral intermediate for agrochemical products and can be used to introduce the chiral centre in APIs and pesticides or it can be a resolving agent itself. ${ }^{10,11}$ Among some other ways to produce its optically active form, it was resolved by diastereomeric salt formation using $(R)$-1phenylethane-1-amine by $\mathrm{He}$ et al. ${ }^{12}$ The melting points of the diastereomeric salts were also presented in the cited paper, however, we got slightly different results.

There have been multiple studies on the behaviour of enantiomeric mixtures during separation processes. The intermolecular interactions between the enantiomers can influence the outcome of enantiomeric enrichment processes like sublimation, kinetic resolution and even achiral phase HPLC. ${ }^{13-15}$ Fogassy et al found that the optical purity of the enantiomers obtained in several diastereomeric resolutions via slow atmospheric crystallisation, shows good correlation with the melting eutectic composition (ee) of the resolving agent or the racemic compound, depending on which one is higher. ${ }^{16}$ It is also known that the recrystallisation based purification of non-racemic enantiomeric mixtures is often limited at the same composition as the melting point eutectic of the compound. ${ }^{17}$

Besides presenting gas antisolvent fractionation as a novel diastereomeric enrichment technique, we aim to investigate whether the atmospheric melting point phase diagram and the high pressure purification results might be correlated.

\section{Materials and Methods}

\section{MATERIALS}

Carbon dioxide (>99.9\%) used in the experiments was purchased from Linde Gas Hungary Co. Cltd. and was used freshly distilled. Racemic 4-chloromandelic acid (CAS 7138-34-3) and (R)-4-chloromandelic acid (CAS 32189-36-9) (purities over 98\%) were purchased from TCl Co. Ltd., while both enantiomers of 1-phenylethylamine (CAS 3886-69-9 for $R$ and 2627-86-3 for $S$ enantiomer, $>99 \%$ ) were purchased from Merck Ltd. Methanol (>99\%) was bought from Molar Chemicals Ltd.

\section{GAS ANTISOLVENT FRACTIONATION AS A FURTHER PURIFICATION METHOD}

4-Chloromandelic acid of the desired enantiomeric excess was prepared by dissolving the mixture of the racemic compound and the pure $(R)$-enantiomer in methanol, then evaporating the solvent. The enantiomeric mixture was dried under atmospheric conditions overnight. Calculated enantiomeric excess was confirmed by chiral capillary electrophoresis.
$100 \mathrm{mg}$ of the prepared non-racemic acid and $64.8 \mathrm{mg}$ of the desired enantiomer of the amine (in molar equivalent quantity) were mixed in a vial, and $4.5 \mathrm{ml}$ of methanol was added. The homogenous solution was then filled into the tempered laboratory autoclave as shown in FIGURE 2. The autoclave was pressurised with supercritical $\mathrm{CO}_{2}$, using a Teledyne ISCO 260D syringe pump with known quantity of $\mathrm{CO}_{2}$ to set a $\mathrm{CO}_{2}$ : methanol mass ratio of 7.5. The mixture was stirred at $16 \mathrm{MPa}, 40^{\circ} \mathrm{C}$ for an hour followed by washing the autoclave with supercritical $\mathrm{CO}_{2}$, equivalent to three times of its volume to extract the dissolved components and the organic solvent. A methanol-filled trap was used to collect the extracted compounds. Upon opening the autoclave, a white, visibly crystalline material could be collected as the raffinate.

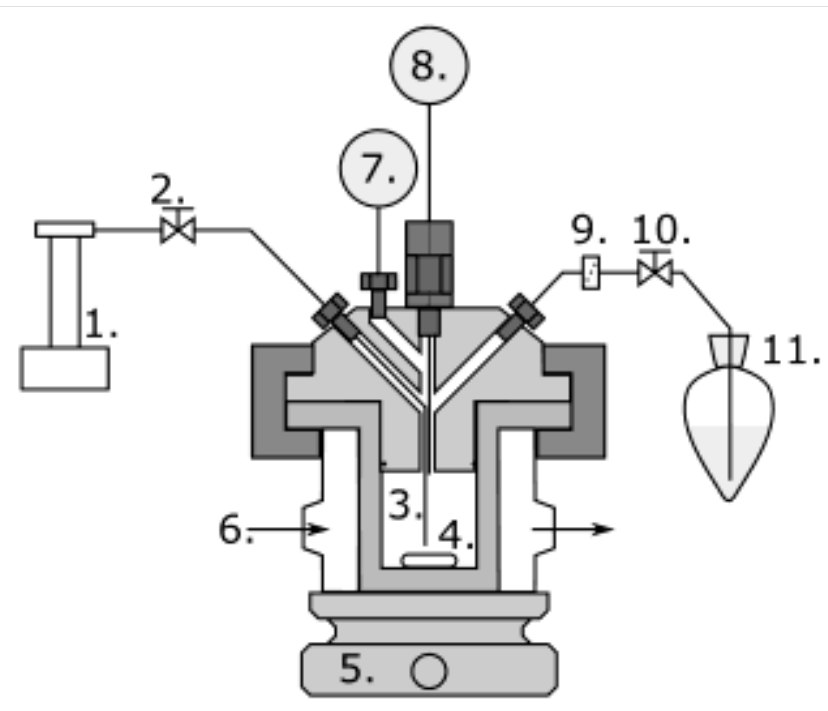

FIGURE 2 Schematic depiction of the laboratory autoclave used in the experiments. 1. ISCO 260D syringe pump; 2. Control valve; 3. Inlet tubing for better mixing; 4. Magnetic stirrer; 5. Magnetic motor; 6. Jacket; 7. Manometer and pressure transducer; 8. Thermocouple; 9. Filter; 10. Control valve; 11. Solvent trap.

As a comparison, recrystallisation experiments were also carried out. In the first step, racemic 4-chloromandelic acid was resolved with a molar equivalent amount of $(R)$-1-phenylethyamine with the GAS method described above. After depressurising the equipment, the raffinate was not collected, only a sample was taken for analytical purposes. Afterwards, the raffinate was again dissolved in the same amount of methanol used before and the GAS process was repeated. Three consecutive crystallisation steps were carried out.

\section{ATMOSPHERIC EXPERIMENTS OF FURTHER PURIFICATION}

$200 \mathrm{mg}$ of enantiomeric mixture of the acid was measured in a test tube together with a molar equivalent quantity of enantiopure amine. The mixture was dissolved in $3.2 \mathrm{ml}$ of methanol at $60^{\circ} \mathrm{C}$ then gradually cooled to $0{ }^{\circ} \mathrm{C}$. The mother liquor was decanted and the crystals were rinsed with $0.5 \mathrm{ml}$ of cold ethanol. Afterwards, the crystalline material was left to dry overnight. 


\section{ANALYTICAL METHODS}

Collected samples and starting materials were analysed by capillary electrophoresis in order to determine the enantiomeric (diastereomeric) composition of the samples. An Agilent 7100 equipment fitted with a $50 \mathrm{~cm}$ long polyimide-covered uncoated silica capillary was used. Experiments were performed in a $\mathrm{pH} 9$, $50 \mathrm{mM}$ borate buffer. Peaks were detected using a DAD detector, with a detection wavelength of $200 \mathrm{~nm}$ and a reference wavelength of $320 \mathrm{~nm}$. Measurements were carried out at $25^{\circ} \mathrm{C}$ with $20 \mathrm{kV}$ voltage applied to the column. To achieve chiral separation, $10 \mathrm{mM}$ of Monoamino-BCD (product of Cyclolab Ltd) was used as a chiral selector. Since the diastereomeric salt dissociates under the circumstances of the measurements, the term enantiomeric excess is suitable to describe the composition of the mandelic acid derivative in the salt.

Melting points of the diastereomeric salts were measured using a TA Instruments 2920 Modulated DSC equipment. Approximately $3 \mathrm{mg}$ of each prepared raffinate sample was filled into an aluminium crucible. A heating rate of $5^{\circ} \mathrm{C} / \mathrm{min}$ up to $220^{\circ} \mathrm{C}$ was applied.

Raffinate samples from the GAS experiments were also examined using powder $\mathrm{X}$-ray diffraction. The measurements were carried out using a PANalytical X'pert Pro $(\Theta / \Theta)$ diffractometer measuring from 4 to $462 \theta$ degrees with a complete scan time of 10 minutes. Samples were examined at a wavelength of $1.5408 \AA$ ( $\mathrm{Cu} \mathrm{Ka})$ and with $40 \mathrm{kV}$ and $30 \mathrm{~mA}$ applied to the X-ray tube.

\section{Results and Discussion}

\section{FURTHER PURIFICATION EXPERIMENTS AND MELTING POINT PHASE EQUILIBRIUM}

The diastereomeric purities of the crystalline products of the further purification experiments together with the melting point phase diagram of the diastereomeric salts (FIGURE 3.) are presented in FIGURE 4. For a better comparison with respect to the eutectic composition and the rate of further purification, a similar scale was applied to both plots. The melting point phase behaviour of the diastereomeric salts has been modelled using the Schröder - van Laar equation ${ }^{18}$ taking into account the remeasured melting points and enthalpies, $143.1{ }^{\circ} \mathrm{C} ; 26.7 \mathrm{~kJ} / \mathrm{mol}$ and $200.6^{\circ} \mathrm{C} ; 44.0 \mathrm{~kJ} / \mathrm{mol}$ of the $(S, R)$ - and $(R, R)$ - diastereomeric salts, respectively.<smiles>C[C@H](N)c1ccccc1</smiles><smiles>C[C@H](N)c1ccccc1</smiles>

FIGURE 3 The more stable (RR) (on the left side) and the less stable $(S R)$ (on the right side) diastereomeric salt.
The results of the DSC melting point measurements, modelling and the results of the GAS further purification experiments all suggest a very similar eutectic composition at approx. $80 \%$ purity of the SR-salt. Upon comparing the results of the further purification experiments and the actual consecutive GAS precipitations (presented in FIGURE 4. as a) circle and b) triangle, respectively), it is clear that, regarding the diastereomeric excess, further purification experiments starting from a prepared mixture of enantiomers sufficiently represent the recrystallisation results. However, it is also visible, that de values obtained by GAS precipitation fall back compared to the atmospheric experiments found in the literature or carried out in our laboratory (FIGURE 4. c), square and d), diamond, respectively).

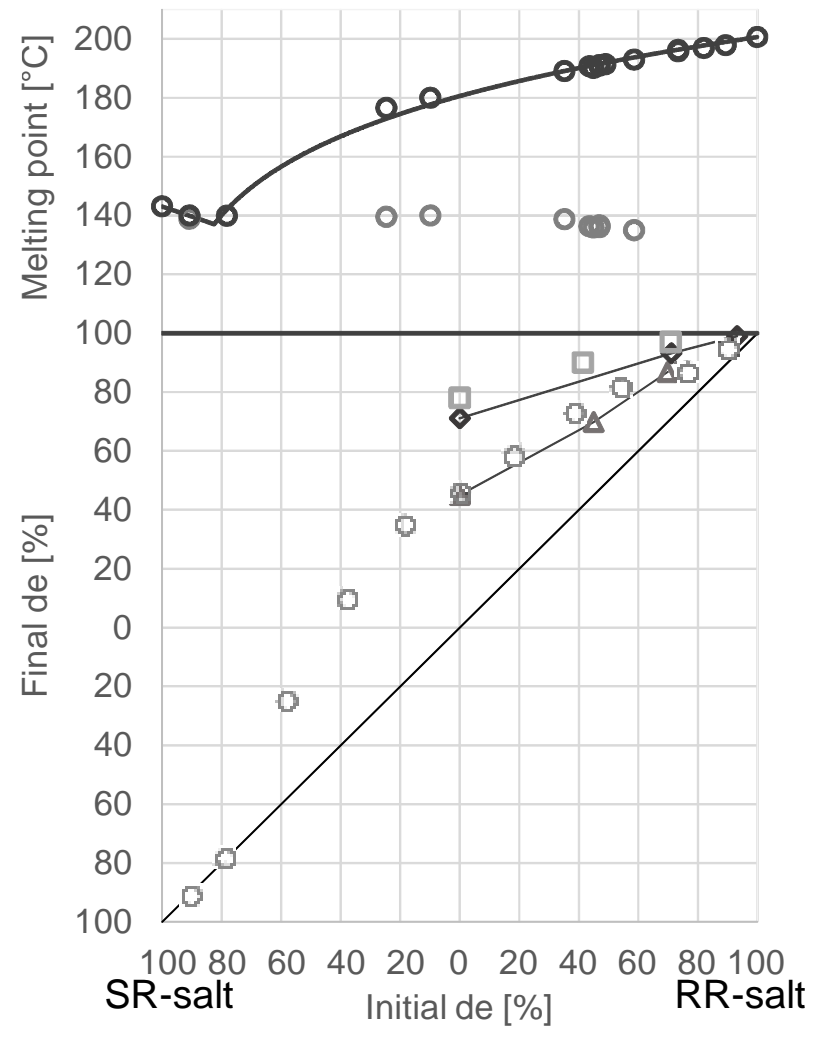

a) $\triangle-$ b) $\square$ c) $\diamond$ d) $\circ$ A) $\circ$ B) - C)

FIGURE 4 Melting point phase diagram and final de vs. initial de diagram of the further purification experiments

a) Further purification experiments by GAS fractionation

b) Actual recrystallisation of GAS raffinate

c) Further purification experiments at atmospheric conditions

d) Actual recrystallisation results obtained from literature ${ }^{12}$

A) Measured eutectic melting points

B) Measured melting points

C) Modelled melting point curve

Comparing the results of consecutive GAS precipitations and the atmospherical reference obtained from the literature (FIGURE 4. b), triangle and d), diamond), one can assume that one more GAS precipitation step would be needed to obtain the same diastereomeric purity.

However, not only the diastereomeric excess is important but yields as well. The optimal further purification results in a large increase of de with maximal yield. The efficiency of diastereomeric 
enrichment ( $E D E$, presented in FIGURE 5.), might be defined analogously to efficiency of enantiomeric enrichment (The efficiency of enantiomeric enrichment $(E E E)$ is defined as the ratio of the enantiomeric purities of the product and the starting material multiplied by the yield. ${ }^{19}$ ). The atmospheric and the GAS fractionation methods have similar efficiencies (FIGURE 5.).

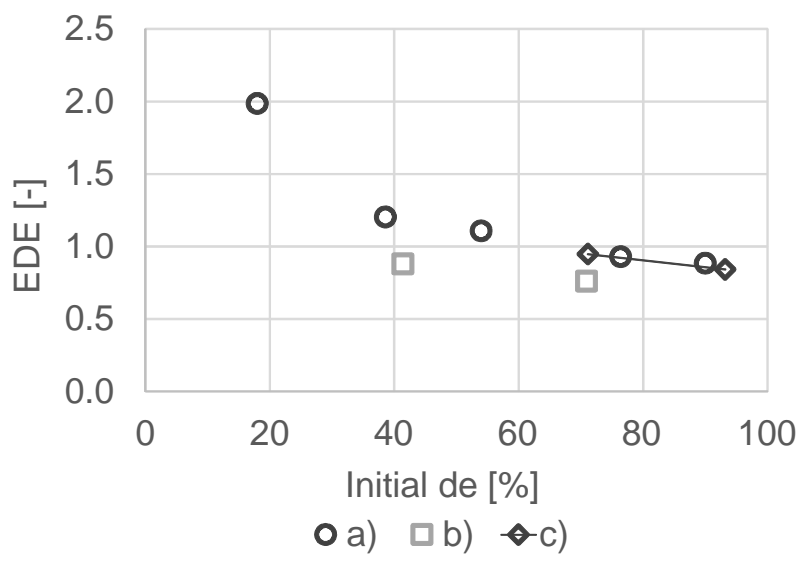

FIGURE 5 Efficiency of diastereomeric enrichment (EDE) plotted against the initial de.

a) GAS experiments on further purification

b) Atmospheric further purification experiments

c) Recrystallisation experiments from the literature ${ }^{12}$

Meanwhile GAS yields slightly exceed the published atmospheric yields and highly overcome the yields of our own atmospheric references. (FIGURE 6.) The increasing trend of the yield with increasing initial diastereomeric excess is due to the higher proportion of the less soluble salt in the solution having the same overall concentration.

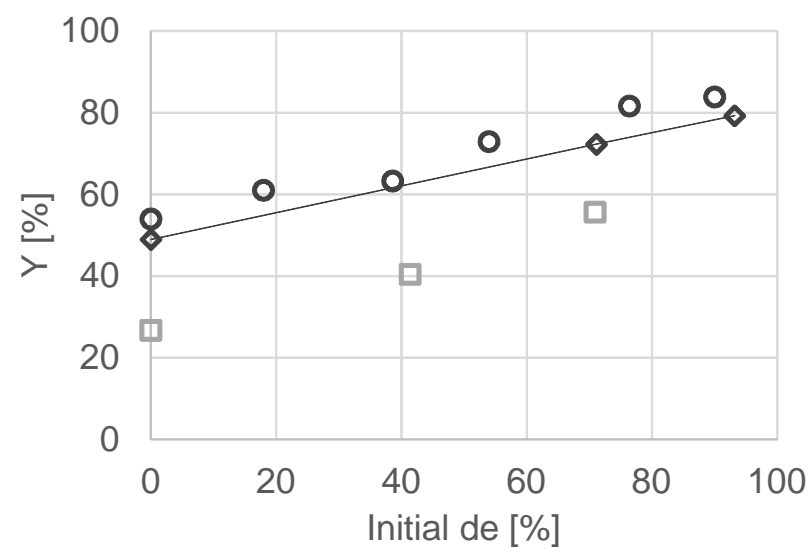

o a) $\square$ b) $\diamond$ c)

FIGURE 6 The mass ratio between the raffinate and the starting material (i. e. the raffinate yield; $Y$ ) plotted against the initial de

a) GAS experiments on further purification

b) Atmospheric further purification experiments

c) Recrystallisation experiments from the literature ${ }^{12}$

\section{SOLID STATE CONFIRMATION OF THE PURIFICATION} PROCESS BY XRD

Powder X-ray diffraction phase analysis was performed on the crystalline samples. The diffractograms of both of the diastereomeric salts and three of the raffinate samples are depicted in FIGURE 6. While b), c) and d) are actual powder samples from this study, a) and b) are calculated powder XRD patterns of $((R)$-1-phenylethanaminium $\quad(S)$-4chloromandelate $)^{20,21}$ and $((R)$-1-phenylethylaminium $(R)$-4chloromandelate $)^{22,23}$, generated from single crystal atomic coordinates of $\mathrm{VIQPUH}^{20,21}$ and $\mathrm{WIPBIH}^{22,23}$, respectively, retrieved from Cambridge Structural Database $(C S D)^{24}$ by Mercury program ${ }^{25}$.

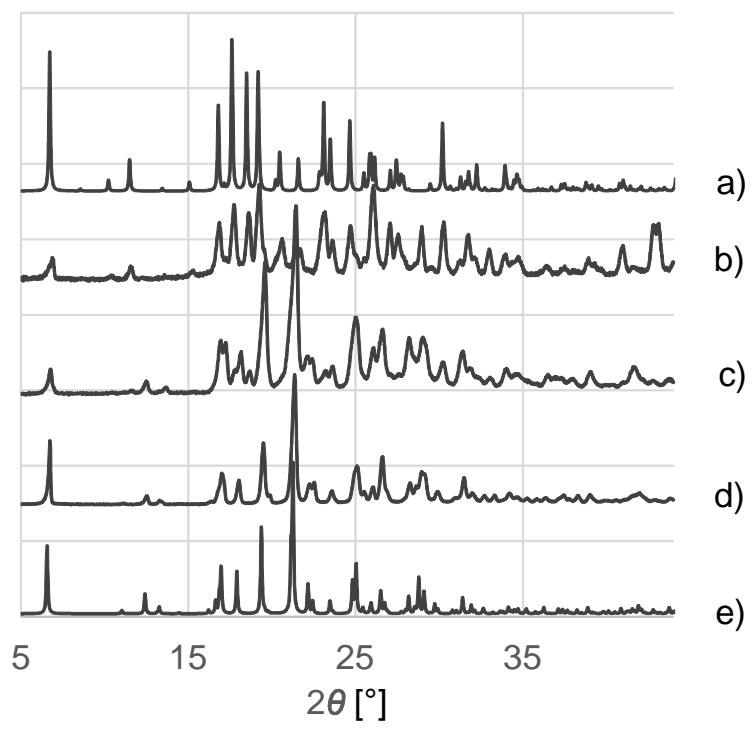

FIGURE 7 Comparison of XRD diffractograms

a) Less stable (R)-acid -(S)-amine salt ${ }^{20,21}$

b) Raffinate of $d e=90 \%$, enriched in (R)-acid - (S)-amine salt

c) Raffinate of $d e=10 \%$, enriched in (R)-acid - (S)-amine salt

d) Raffinate of $d e=89 \%$, enriched in $(\mathrm{R})$-acid - (R)-amine salt

e) More stable (R)-acid - (R)-amine salt ${ }^{22,23}$

Comparing the diffraction profiles of the obtained crystalline products, as it is expectable, the higher de salts (FIGURE 7. b) and d)) show similar diffraction patterns to those of diastereomeric salts formed from the enantiomers they are enriched in (i. e. a) and e), respectively). The sample c), which is close to the salt formed from a racemic acid, appears to be a mixture of the two diastereomers. It can also be concluded, that the precipitation under high pressure does not change the crystal structure of the diastereomeric salts.

\section{Conclusion}

In this case study we investigated the possibility of the further purification of 4-chloromandelic acid enantiomers by the recrystallisation of their $(R)$-1-phenylethanaminium salts. The gas antisolvent fractionation is a fast, reliable and efficient way to increase the diastereomeric purity of the salts. The binary melting 
point phase diagram and the $d e_{1}-d e_{0}$ diagram show a eutectic point at the same de value. It is a slightly counter-intuitive result, because the very quick precipitation occurring under the circumstances of GAS fractionation, in contrast with the slow traditional crystallisation controlled by a cooling program, suggest that kinetic effects should be dominant.

GAS can be an alternative to traditional resolution and enantiomeric further purification methods. Efficiency of diastereomeric enrichment values, hence the efficiency of the further purification are very similar, which in the case of the GAS experiments could probably even be improved by some parameter optimisation and upscaling. This, and some other advantages of the anti-solvent techniques - practically solventfree products, the possibility of controlling the particle size and its distribution, the usage of less organic solvents - suggest that they could be efficient tools in producing enantiopure compounds.

\section{Acknowledgements}

We highly appreciate the fruitful discussions with Prof. Elemér Fogassy and the assistance of Klára Sai-Halász in the capillary electrophoretic measurements. Our research work was supported by OTKA (K108979). E. Sz. thanks to the support provided by the Hungarian Academy of Sciences through the Bolyai Scholarship for Researchers.

\section{REFERENCES}

1. Fogassy E, Ács M, Szili T, Simándi B, Sawinsky J. Molecular chiral recognition in supercritical solvents. Tetrahedron Lett 1994;35:257-260.

2. Keszei S, Simandi B, Fogassy E, Sawinsky J, Niklos C, Lovas R. Resolution of ibuprofen and cis-chrysanthemic acid by supercritical fluid extraction. HIGH Press Chem Eng 1996;12:393-398.

3. Bánsághi G, Székely E, Sevillano DM, Juvancz Z, Simándi B. Diastereomer salt formation of ibuprofen in supercritical carbon dioxide. J Supercrit Fluids 2012;69:113-116.

4. Bánsághi G, Lőrincz L, Szilágyi IM, Madarász J, Székely E. Crystallization and Resolution of cis-Permethric Acid with Carbon Dioxide Antisolvent. Chem Eng Technol 2014;37:1417-1421.

5. Martín A, Cocero MJJ. Separation of enantiomers by diastereomeric salt formation and precipitation in supercritical carbon dioxide. J Supercrit Fluids 2007;40:6773.

6. Esfandiari N. Production of micro and nano particles of pharmaceutical by supercritical carbon dioxide. J Supercrit Fluids 2015;100:129-141.

7. Martín V, Romero-Díez R, Rodríguez-Rojo S, Cocero MJ. Titanium dioxide nanoparticle coating in fluidized bed via supercritical anti-solvent process (SAS). Chem Eng J 2015;279:425-432.

8. Montes A, Kin N, Gordillo MD, Pereyra C, De La Ossa EJM. Polymer-naproxen precipitation by supercritical antisolvent (SAS) process. J Supercrit Fluids 2014;89:58-67.

9. Pasquali I, Bettini R. Are pharmaceutics really going supercritical? Int J Pharm 2008;364:176-187.
10. Liu X, Yi Z, Wang J, Liu G. Decarboxylative acylation of arenes with mandelic acid derivatives via palladiumcatalyzed oxidative sp $2 \mathrm{C}-\mathrm{H}$ activation. $R S C \mathrm{Adv}$ 2015;5:10641-10646.

11. Tashiro Y, Nagashima T, Aoki S, Aboshi Y. Optically active complex, alanine.ring-substituted mandelic acid, and the method for producing the same. 1983;86.

12. Quan HE, Peng YF, Rohani S. Diastereomeric resolution of p-chloromandelic acid with (R)-phenylethylamine. Chirality 2010;22:16-23.

13. Perry RH, Wu C, Nefliu M, Cooks RG. Serine sublimes with spontaneous chiral amplification. Chem Commun 2007;0:1071-1073.

14. Satyanarayana T, Kagan HB. Amplification of the enantiomeric excess of a compound in kinetic resolution by a racemic reagent. Tetrahedron 2007;63:6415-6422.

15. Soloshonok VA. Remarkable amplification of the selfdisproportionation of enantiomers on achiral-phase chromatography columns. Angew Chemie - Int Ed 2006;45:766-769.

16. Pálovics E, Szeleczky Z, Fődi BB, Faigl F, Fogassy E. Prediction of the efficiency of diastereoisomer separation on the basis of the behaviour of enantiomeric mixtures. RSC Adv 2014;4:21254-21261.

17. Faigl F, Fogassy E, Nógrádi M, Pálovics E, Schindler J. Separation of non-racemic mixtures of enantiomers: an essential part of optical resolution. Org Biomol Chem 2010;8:947-959.

18. He Q, Zhu J, Gomaa H, Jennings M, Rohani S. Identification and characterization of solid-state nature of 2chloromandelic acid. J Pharm Sci 2009;98:1835-1844.

19. Fogassy E, Nógrádi M, Kozma D, Egri G, Pálovics E, Kiss V. Optical resolution methods. Org Biomol Chem 2006;4:3011-3030.

20. He Q, Jennings MC, Rohani S, Zhu J, Gomaa H. (R)-1Phenylethanaminium (S)-4-chloromandelate. Acta Crystallogr Sect E Struct Reports Online 2008;64:05590559.

21. He Q, Jennings MC, Rohani S, Zhu J, Gomaa H. (R)-1Phenylethylaminium (S)-4-chloromandelate (VIQPUH; CCDC 680635). Exp Cryst Struct Determ 2008 DOI: $10.5517 /$ ccqv7zr

22. He Q, Jennings MC, Rohani S, Zhu J, Gomaa H. ( R )Phenylethylammonium ( R )-4-chloromandelate. Acta Crystallogr Sect E Struct Reports Online 2007;63:0419904199.

23. He Q, Jennings MC, Rohani S, Zhu J, Gomaa H. (R)-1phenylethylaminium (R)-4-chloromandelate (WIPBIH; CCDC 667265). Exp Cryst Struct Determ 2007 DOI: $10.5517 /$ ccqdbp3

24. Allen FH. The Cambridge Structural Database: a quarter of a million crystal structures and rising. Acta Crystallogr Sect B Struct Sci 2002;58:380-388.

25. Macrae CF, Bruno IJ, Chisholm JA, Edgington PR, McCabe P, Pidcock E, Rodriguez-Monge L, Taylor R, van de Streek J, Wood PA. Mercury CSD 2.0 - new features for the visualization and investigation of crystal structures. J Appl Crystallogr 2008;41:466-470. 
Chirality 